\title{
Factors associated with long-term care certification in older adults: a cross- sectional study based on a nationally representative survey in Japan
}

Akira Momose ${ }^{1,2}$, Satoko Yamaguchi ${ }^{1 *+}$, Akira Okada ${ }^{1}$, Kayo Ikeda-Kurakawa ${ }^{1,2}$, Daisuke Namiki ${ }^{1,2}$, Yasuhito Nannya ${ }^{3}$, Hideki Kato ${ }^{1,4}$, Toshimasa Yamauchi ${ }^{5}$, Masaomi Nangaku ${ }^{4}$ and Takashi Kadowaki ${ }^{1,5,6+}$

\begin{abstract}
Background: Long-term care (LTC) prevention is a pressing concern in ageing societies. To understand the risk factors of LTC, it is vital to consider psychological and social factors in addition to physical factors. Owing to a lack of relevant data, we aimed to investigate the social, physical and psychological factors associated with LTC using large-scale, nationally representative data to identify a high-risk population for LTC in terms of multidimensional frailty.

Methods: We performed a cross-sectional study using anonymised data from the 2013 Comprehensive Survey of Living Conditions conducted by the Ministry of Health, Labour and Welfare of Japan. Among the 23,730 eligible people aged 65 years or older and those who were not in hospitals or care facilities during the survey, 1718 stated that they had LTC certification. Univariate and multivariate logistic regression analyses were performed to determine the factors associated with LTC certification.

Results: Factors positively associated with LTC certification in the multivariate analyses included older age, the interaction term between sex and age group at age 85-89 years, limb movement difficulties, swollen/heavy feet, incontinence, severe psychological distress (indicated by a Kessler Psychological Distress Scale [K6] score $\geq 13$ ), regular hospital visits for dementia, stroke, Parkinson's disease, chronic obstructive pulmonary disease, fracture, rheumatoid arthritis, kidney disease, diabetes and osteoporosis. Factors negatively associated with LTC certification included the presence of a spouse, regular hospital visits for hypertension and consulting with friends or acquaintances about worries and stress.

Conclusions: In summary, we identified the physical, psychological and social factors associated with LTC certification using nationally representative data. Our findings highlight the importance of the establishment of multidimensional approaches for LTC prevention in older adults.
\end{abstract}

Keywords: Long-term care, Frailty, Psychological distress, Social interaction

\footnotetext{
* Correspondence: syamaguc-tky@umin.ac.jp

†'Satoko Yamaguchi and Takashi Kadowaki contributed equally to this work.

'Department of Prevention of Diabetes and Lifestyle-Related Diseases,

Graduate School of Medicine, The University of Tokyo, 7-3-1 Hongo,

Bunkyo-ku, Tokyo 113-8655, Japan

Full list of author information is available at the end of the article
}

(c) The Author(s). 2021 Open Access This article is licensed under a Creative Commons Attribution 4.0 International License, which permits use, sharing, adaptation, distribution and reproduction in any medium or format, as long as you give appropriate credit to the original author(s) and the source, provide a link to the Creative Commons licence, and indicate if changes were made. The images or other third party material in this article are included in the article's Creative Commons licence, unless indicated otherwise in a credit line to the material. If material is not included in the article's Creative Commons licence and your intended use is not permitted by statutory regulation or exceeds the permitted use, you will need to obtain permission directly from the copyright holder. To view a copy of this licence, visit http://creativecommons.org/licenses/by/4.0/ The Creative Commons Public Domain Dedication waiver (http://creativecommons.org/publicdomain/zero/1.0/) applies to the data made available in this article, unless otherwise stated in a credit line to the data. 


\section{Background}

Population ageing is a growing concern, worldwide. In Japan, the country with the highest proportion of elderly citizens, $28.4 \%$ of the population was aged $\geq 65$ years in 2019. The public long-term care insurance system was introduced in 2000 in Japan to accommodate the growing long-term care (LTC) needs [1]. People aged 65 years or older are eligible for LTC irrespective of the reason, while people aged 40-64 years are entitled to it only for certain age-related diseases. The eligibility for LTC is assessed using a standardised 74-item questionnaire based on activities of daily living (ADLs) and a physician's report. Availability of family caregiving and household income are not considered when determining eligibility [2]. There exist five levels of LTC certification, care levels 1 to 5 (most severe disability), depending on the ADL. In addition, support levels 1 and 2 are meant for people who are eligible for LTC prevention services. The certification rates in people aged $\geq 65$ years were 13.5\% (care levels 1-5) and 5.2\% (support levels 1-2) as of April 2020 [3]. Even though the eligibility criteria are uniform nationwide, LTC certification rates vary by region after adjusting for age [4]. Insurance benefits include in-home services (e.g., home visits, day services and short-stay services), services at care facilities and community-based services, but they do not include cash benefits or other direct benefits for family caregivers [2]. All services are subject to $10-30 \%$ co-payment, depending on the income. Approximately $90 \%$ of people with care levels 1-5 certification utilised the services in April 2020 [3]. Most privately funded LTC insurances complement the public LTC system by providing cash benefits for those who have obtained public LTC certification.

Identifying high-risk populations for LTC and mitigating the need for LTC is crucial for the extension of a healthy life expectancy. Recently, the concept of frailty has attracted a high degree of attention in this context, as frail people have an increased risk of LTC and mortality [5]. Although the concept was originally developed predominantly in terms of physical function, its multidimensional nature has been largely recognised. It is characterised by a decline in one or more domains of human functioning, including the physical, psychological and social domains [6]. Therefore, to understand the risks associated with LTC needs, social and psychological factors must be taken into consideration in addition to physical or clinical factors.

The physical and clinical factors associated with LTC have been investigated extensively. Studies using medical and LTC claims data in Japan and Germany identified chronic conditions associated with LTC certification, including fractures, dementia, pneumonia, strokes, Parkinson's disease, diabetes and arthropathy [7-9]. According to the Comprehensive Survey of Living Conditions
(CSLC) in Japan, the major causes of LTC certification are stroke, dementia and infirmity to ageing [10].

In addition to these factors, psychological and social factors also play an important role. The components of psychological frailty include cognitive impairment and depressive symptoms [6]. Depressive symptoms are associated with a subsequent decline in the activities of daily living $[11,12]$ and cognitive function [13].

As for social factors, older age has been univocally identified as a risk factor for LTC. While not having a partner was identified as a risk factor across several studies, reports on the association of sex, education, or socioeconomic status with LTC are less consistent [14-17].

Few studies have considered physical, psychological and social factors simultaneously and those studies included regional cohorts or a relatively small number of participants. Schnitzer et al. reported that care dependency was significantly associated with older age, urinary incontinence, stroke, falls, cancer, diabetes, education level, limited mobility and limited physical activity in a cohort study of 1699 participants aged $\geq 70$ years in Germany [14]. Wu et al. analysed data on 2608 people aged $\geq 65$ years old from the National Health Interview Survey in Taiwan and reported that age, urban living, stroke, dementia and ADL disability were significantly associated with LTC use [16].

We aimed to investigate the social, physical and psychological factors associated with LTC in a large-scale, nationally representative sample, utilising anonymised 2013 CSLC data to identify a high-risk population for LTC in terms of multidimensional frailty.

\section{Methods}

Data sources

Anonymised data from the 2013 CSLC, which became available in September 2018 from the Ministry of Health, Labour and Welfare (MHLW) of Japan, were obtained. Approval to use these data was obtained from the MHLW under Article 36 of the Statistics Law of Japan. The results reported in this study are based on analyses that we performed using anonymised data from the MHLW.

The CSLC is a nation-wide survey conducted by MHLW every 3 years in Japan for the investigation of basic living condition parameters such as health, medical care, welfare, pension and income [18]. In the 2013 CSLC, the household questionnaire and health questionnaire covered $\sim 300,000$ households and 740,000 household members across 5530 districts that were randomly sampled from the National Census in 2010 [10]. Completed self-administered questionnaires were collected by survey takers. The response rate for the household questionnaire and health questionnaire was $79.4 \%$ [10]. 
We obtained anonymised data covering 97,345 household members from the household questionnaire and health questionnaire.

\section{Study participants}

The eligibility criteria for participation were age 40 years or older. People who answered 'yes' to the question 'Are you currently in a hospital or care facility?' as well as those who did not answer this question were excluded from the analyses, as their health questionnaires were unavailable.

\section{Variables}

Participants who answered 'yes' to the question 'Do you need assistance or supervision due to disabilities or impaired physical function?' were asked about their LTC certification status. LTC certification status was considered as a dependent variable and all the others as independent variables.

Independent variables were categorised into three, according to the Andersen Model [19], which is widely used to explain health-care utilisation: 1) predisposing factors, 2) enabling factors and 3) need factors. The independent variables used in the present study are as follows: 1) predisposing factors: age groups (65-69, 70-74, $75-79,80-84,85-89, \geq 90$ years), sex and education level ( $>9$ or $\leq 9$ years); 2 ) enabling factors: equivalent disposable income as calculated by dividing the household disposable income by the square root of the number of household members ( $\geq 100,000$ or $<100,000$ yen), type of housing (owned or rented), presence of a spouse, household structure (single/couple-only households vs other types of households [e.g., households with parent(s) and child(ren), three-generation households, etc]) and presence of children living separately; 3) need factors: Subjective symptoms: the participants were asked if they had experienced any subjective symptoms in the last several days; Regular hospital visits: the participants were asked if they were regularly visiting hospitals, clinics or therapists for any disease or injuries; Persons with whom the participants discussed their worries and stress, if applicable; and the Kessler Psychological Distress Scale (K6). The K6 comprises six questions pertaining to the assessment of psychological distress and is widely used to screen for depression and anxiety [20]. A K6 score $\geq$ 13 indicates severe psychological distress [20]. Variables that were likely to be the results of the conditions that need LTC such as employment status or whether they had routine medical check-up were not included in the analyses. Information on regions or medication were unavailable. Correlation coefficients between the two independent variables were calculated by Spearman's rank correlation test; for pairs with correlations coefficients >
0.4 or $<-0.4$, the less representative variable was excluded.

Participants who answered 'yes' to the question 'Do you need assistance or supervision due to disabilities or impaired physical function?' answered additional questions about their degree of independence in daily life activities. The anonymised data did not include information on certified care need level (levels 1-5, with levels 3-5 indicating severe need). Instead, we evaluated the severity of care needs based on the 'degree of independence in the daily life activities'. Certified participants who answered 'I spend all day in bed and need assistance in the toilet, in eating and in dressing' or 'I need help at home and spend more time in bed but can maintain a sitting position' were defined as having 'a lower degree of independence', whereas those who answered, 'I am largely independent at home but need help when I go out' or 'I have some disabilities but am largely independent in daily life and can go out alone' were considered to have 'a higher degree of independence'. The sensitivity and specificity of 'lower degree of independence' based on this criterion for the identification of people with care levels 3-5, calculated based on open 2013 CSLC data [18] are 72.8 and $77.8 \%$, respectively.

\section{Statistical analysis}

The associations between LTC certification and the independent variables were evaluated using univariate logistic regression analyses.

To construct a model using a training dataset and validate it using a testing dataset, the data were split randomly into a training dataset and testing dataset at a ratio of 4:1 before multivariate analyses were performed. For the training dataset, the multiple imputation method was applied to fill missing values. We prepared $20 \mathrm{im}$ puted datasets by multiple imputation employing the chained equation using mice package in $\mathrm{R}$ [21]. Multivariate logistic regression models were built by combining the estimates obtained from the 20 imputed datasets using Rubin's rules. Variable selection was performed by stepwise model selection using the Akaike information criterion in each dataset. The variables that were selected in at least 11 datasets were included in the final models. The final models were built by combining the estimates obtained from 20 imputed datasets using Rubin's rules.

Receiver operating characteristic (ROC) curves were drawn by adapting the models to the testing dataset and the area under the curve (AUC) was calculated.

We evaluated differences between certified participants with 'a lower degree of independence in daily life activities' and those with 'a higher degree of independence in daily life activities', and differences between the participants with or without LTC certification among those 
who needed assistance or supervision due to disabilities or impaired physical function. Non-adjusted odds ratios were determined using univariate logistic regression analyses. To determine the adjusted odds ratios of factors associated with LTC certification indicating a lower or higher degree of independence, we excluded 147 certified participants whose degrees of independence were unknown. Multivariate analyses were performed by comparing certified participants with a lower degree of independence with the other participants or those with a higher degree of independence with non-certified participants. A two-sided $p$ value $<0.05$ was considered statistically significant. All the statistical analyses were performed using R, version 4.0.2 (The R Foundation for Statistical Computing, Vienna, Austria) and STATA, version 15 (STATACorp LLC., Texas, USA).

\section{Results}

The anonymised data of 97,345 participants were obtained. Of 58,971 people who were aged $\geq 40$ years, 1128 who answered 'yes' to the question 'Are you currently in a hospital or care facility?' and 895 who did not answer the question were excluded. The remaining 56,948 people were considered eligible for participation (Fig. 1).
We predominantly analysed data on participants aged $\geq 65$ years, as people aged $40-64$ years can receive LTC certification only if they have one of 16 age-related diseases. Of 23,730 participants aged $\geq 65$ years, 1718 (7.2\%) were certified.

The basic characteristics of the participants aged $\geq 65$ years with and without LTC certification are shown in Table 1 . The certification rate was only $1.6 \%$ in the $65-$ 69 years' age group but as high as $45.0 \%$ in the $\geq 90$ years age group.

Univariate logistic regression was performed to evaluate the association between LTC certification and the independent variables in total, in men and in women (Table 2). In total, women were more likely than men to have LTC certification (odds ratio [OR] 1.74, 95\% confidence interval $[\mathrm{CI}]$ 1.56-1.93). The older age groups were strongly associated with LTC certification. The increase in the OR with age was more enhanced in the women than in men; the ORs in the $\geq 90$ years' age group compared to those in the 65-69 age group were 26.22 in men and 63.95 in women.

The adjusted odds ratios (aOR) obtained in the multivariate logistic regression analysis using the imputed training dataset are shown in Table 3. As age had a

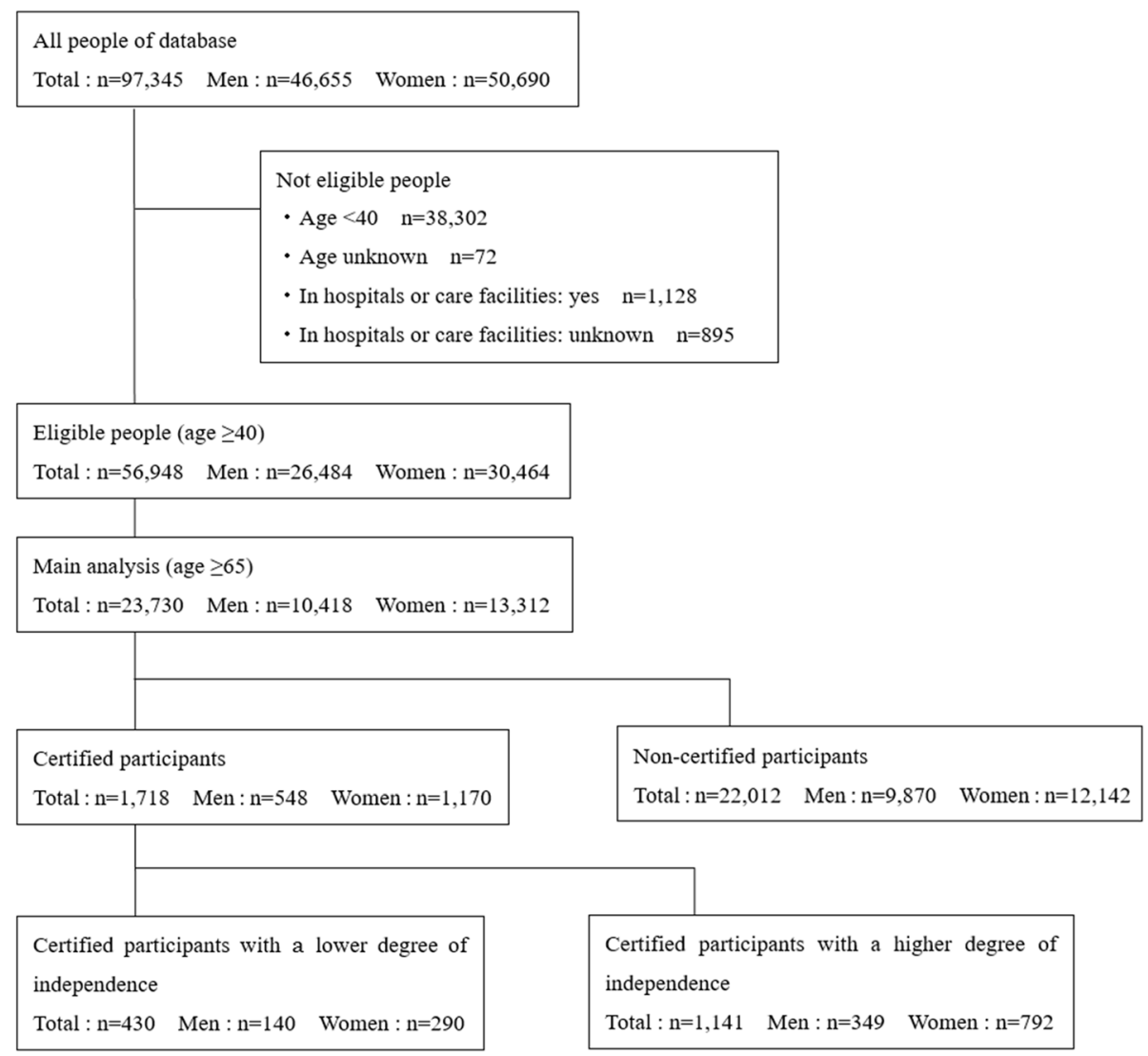

Fig. 1 Flowchart of the study participant selection process 
Table 1 Basic characteristics of participants aged $\geq 65$ years with and without LTC certification

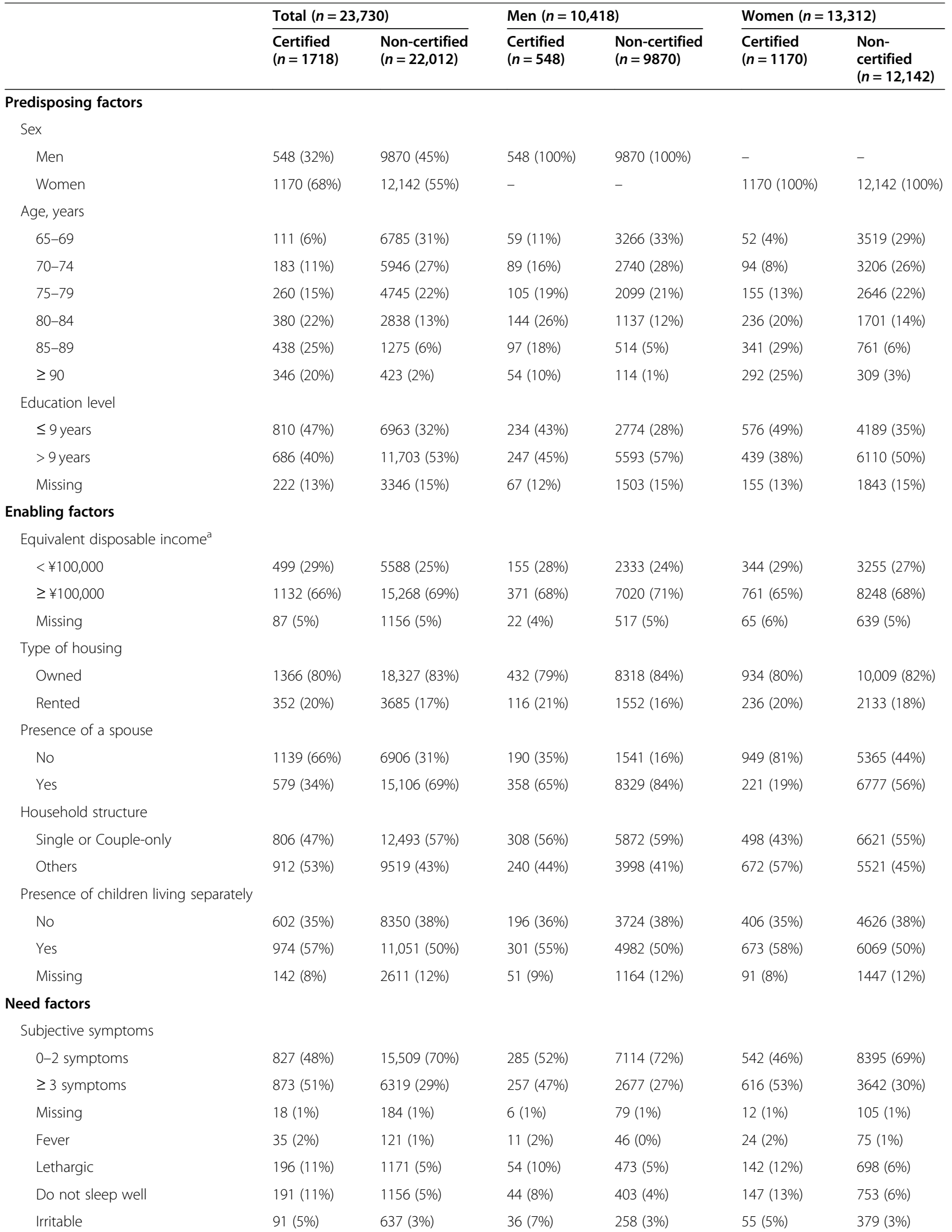


Table 1 Basic characteristics of participants aged $\geq 65$ years with and without LTC certification (Continued)

\begin{tabular}{|c|c|c|c|c|c|c|}
\hline & \multicolumn{2}{|c|}{ Total $(n=23,730)$} & \multicolumn{2}{|c|}{ Men $(n=10,418)$} & \multicolumn{2}{|c|}{ Women $(n=13,312)$} \\
\hline & $\begin{array}{l}\text { Certified } \\
(n=1718)\end{array}$ & $\begin{array}{l}\text { Non-certified } \\
(n=22,012)\end{array}$ & $\begin{array}{l}\text { Certified } \\
(n=548)\end{array}$ & $\begin{array}{l}\text { Non-certified } \\
(n=9870)\end{array}$ & $\begin{array}{l}\text { Certified } \\
(n=1170)\end{array}$ & $\begin{array}{l}\text { Non- } \\
\text { certified } \\
(n=12,142)\end{array}$ \\
\hline Forgetful & $422(25 \%)$ & 1916 (9\%) & $111(20 \%)$ & $773(8 \%)$ & 311 (27\%) & $1143(9 \%)$ \\
\hline Headache & $102(6 \%)$ & $679(3 \%)$ & $20(4 \%)$ & $197(2 \%)$ & $82(7 \%)$ & $482(4 \%)$ \\
\hline Dizziness & $128(7 \%)$ & $806(4 \%)$ & $34(6 \%)$ & 287 (3\%) & $94(8 \%)$ & $519(4 \%)$ \\
\hline Blurred vision & $273(16 \%)$ & $2256(10 \%)$ & 79 (14\%) & $884(9 \%)$ & $194(17 \%)$ & $1372(11 \%)$ \\
\hline Difficulty in seeing & $283(16 \%)$ & 1649 (7\%) & $84(15 \%)$ & $661(7 \%)$ & 199 (17\%) & $988(8 \%)$ \\
\hline Ringing ears & $113(7 \%)$ & $1564(7 \%)$ & $37(7 \%)$ & $735(7 \%)$ & $76(6 \%)$ & $829(7 \%)$ \\
\hline Difficulty in hearing & 355 (21\%) & 2015 (9\%) & $89(16 \%)$ & 935 (9\%) & $266(23 \%)$ & $1080(9 \%)$ \\
\hline Palpitations & $123(7 \%)$ & $770(3 \%)$ & $27(5 \%)$ & $318(3 \%)$ & $96(8 \%)$ & $452(4 \%)$ \\
\hline Short-winded & $154(9 \%)$ & $945(4 \%)$ & $56(10 \%)$ & $496(5 \%)$ & $98(8 \%)$ & 449 (4\%) \\
\hline Pain in chest & $59(3 \%)$ & $366(2 \%)$ & $14(3 \%)$ & $161(2 \%)$ & $45(4 \%)$ & $205(2 \%)$ \\
\hline Cough, phlegmatic & $242(14 \%)$ & $1478(7 \%)$ & $107(20 \%)$ & $779(8 \%)$ & $135(12 \%)$ & $699(6 \%)$ \\
\hline Blocked/runny nose & $130(8 \%)$ & 1108 (5\%) & $49(9 \%)$ & $595(6 \%)$ & $81(7 \%)$ & $513(4 \%)$ \\
\hline Wheezing & $87(5 \%)$ & $356(2 \%)$ & $39(7 \%)$ & $193(2 \%)$ & $48(4 \%)$ & $163(1 \%)$ \\
\hline Stomach upset/heartburn & $90(5 \%)$ & $986(4 \%)$ & $24(4 \%)$ & $406(4 \%)$ & $66(6 \%)$ & $580(5 \%)$ \\
\hline Diarrhoea & $84(5 \%)$ & $376(2 \%)$ & $28(5 \%)$ & $206(2 \%)$ & $56(5 \%)$ & $170(1 \%)$ \\
\hline Constipation & $277(16 \%)$ & $1729(8 \%)$ & $91(17 \%)$ & $723(7 \%)$ & $186(16 \%)$ & $1006(8 \%)$ \\
\hline Loss of appetite & $97(6 \%)$ & $346(2 \%)$ & $29(5 \%)$ & $156(2 \%)$ & $68(6 \%)$ & $190(2 \%)$ \\
\hline Abdominal pain/stomachache & $63(4 \%)$ & $377(2 \%)$ & $22(4 \%)$ & $154(2 \%)$ & $41(4 \%)$ & $223(2 \%)$ \\
\hline Painful/bleeding hemorrhoids & $38(2 \%)$ & $293(1 \%)$ & $17(3 \%)$ & $163(2 \%)$ & $21(2 \%)$ & $130(1 \%)$ \\
\hline Toothache & $54(3 \%)$ & $573(3 \%)$ & $19(3 \%)$ & $269(3 \%)$ & $35(3 \%)$ & $304(3 \%)$ \\
\hline Swollen/bleeding gums & $68(4 \%)$ & $680(3 \%)$ & $23(4 \%)$ & $304(3 \%)$ & $45(4 \%)$ & $376(3 \%)$ \\
\hline Difficulty in chewing & $196(11 \%)$ & $1162(5 \%)$ & $58(11 \%)$ & $501(5 \%)$ & $138(12 \%)$ & $661(5 \%)$ \\
\hline Rash & $59(3 \%)$ & $389(2 \%)$ & $20(4 \%)$ & $180(2 \%)$ & $39(3 \%)$ & $209(2 \%)$ \\
\hline Itching & $188(11 \%)$ & $1319(6 \%)$ & $74(14 \%)$ & 727 (7\%) & $114(10 \%)$ & $592(5 \%)$ \\
\hline Joint pain in hands/feet & $388(23 \%)$ & $2730(12 \%)$ & $88(16 \%)$ & $939(10 \%)$ & $300(26 \%)$ & $1791(15 \%)$ \\
\hline Difficulty in limb movement & $518(30 \%)$ & $1542(7 \%)$ & $160(29 \%)$ & $555(6 \%)$ & $358(31 \%)$ & $987(8 \%)$ \\
\hline Numb limbs & $301(18 \%)$ & $1708(8 \%)$ & $100(18 \%)$ & $758(8 \%)$ & $201(17 \%)$ & $950(8 \%)$ \\
\hline Cold limbs & $251(15 \%)$ & $1242(6 \%)$ & $73(13 \%)$ & $431(4 \%)$ & $178(15 \%)$ & $811(7 \%)$ \\
\hline Swollen/heavy feet & $312(18 \%)$ & $1200(5 \%)$ & $79(14 \%)$ & $348(4 \%)$ & $233(20 \%)$ & $852(7 \%)$ \\
\hline Difficulty in/painful urination & $82(5 \%)$ & $509(2 \%)$ & $43(8 \%)$ & $386(4 \%)$ & $39(3 \%)$ & $123(1 \%)$ \\
\hline Frequent urination & 240 (14\%) & $1629(7 \%)$ & $87(16 \%)$ & $974(10 \%)$ & $153(13 \%)$ & $655(5 \%)$ \\
\hline Incontinence & $227(13 \%)$ & $629(3 \%)$ & $60(11 \%)$ & $218(2 \%)$ & $167(14 \%)$ & $411(3 \%)$ \\
\hline Injury including cut, burn & $20(1 \%)$ & $128(1 \%)$ & $4(1 \%)$ & $60(1 \%)$ & $16(1 \%)$ & $68(1 \%)$ \\
\hline \multicolumn{7}{|l|}{ Regular hospital visits } \\
\hline $0-2$ diseases & 1009 (59\%) & 16,860 (77\%) & 325 (59\%) & 7635 (77\%) & $684(58 \%)$ & 9225 (76\%) \\
\hline$\geq 3$ diseases & 697 (41\%) & $4935(22 \%)$ & $217(40 \%)$ & $2148(22 \%)$ & 480 (41\%) & 2787 (23\%) \\
\hline Missing & $12(1 \%)$ & $217(1 \%)$ & $6(1 \%)$ & $87(1 \%)$ & $6(1 \%)$ & $130(1 \%)$ \\
\hline Diabetes & $253(15 \%)$ & $2325(11 \%)$ & $112(20 \%)$ & $1298(13 \%)$ & $141(12 \%)$ & $1027(8 \%)$ \\
\hline Obesity & $21(1 \%)$ & $235(1 \%)$ & $7(1 \%)$ & $88(1 \%)$ & $14(1 \%)$ & $147(1 \%)$ \\
\hline Hyperlipidemia & $136(8 \%)$ & $2320(11 \%)$ & $38(7 \%)$ & $685(7 \%)$ & $98(8 \%)$ & 1635 (13\%) \\
\hline Thyroid disease & $41(2 \%)$ & $413(2 \%)$ & $11(2 \%)$ & $68(1 \%)$ & $30(3 \%)$ & 345 (3\%) \\
\hline Mental illness & $55(3 \%)$ & $274(1 \%)$ & $10(2 \%)$ & $76(1 \%)$ & $45(4 \%)$ & $198(2 \%)$ \\
\hline
\end{tabular}


Table 1 Basic characteristics of participants aged $\geq 65$ years with and without LTC certification (Continued)

\begin{tabular}{|c|c|c|c|c|c|c|}
\hline & \multicolumn{2}{|c|}{ Total $(n=23,730)$} & \multicolumn{2}{|c|}{ Men $(n=10,418)$} & \multicolumn{2}{|c|}{ Women $(n=13,312)$} \\
\hline & $\begin{array}{l}\text { Certified } \\
(n=1718)\end{array}$ & $\begin{array}{l}\text { Non-certified } \\
(n=22,012)\end{array}$ & $\begin{array}{l}\text { Certified } \\
(n=548)\end{array}$ & $\begin{array}{l}\text { Non-certified } \\
(n=9870)\end{array}$ & $\begin{array}{l}\text { Certified } \\
(n=1170)\end{array}$ & $\begin{array}{l}\text { Non- } \\
\text { certified } \\
(n=12,142)\end{array}$ \\
\hline Dementia & $316(18 \%)$ & $173(1 \%)$ & $92(17 \%)$ & 75 (1\%) & 224 (19\%) & $98(1 \%)$ \\
\hline Parkinson's disease & $57(3 \%)$ & $80(0 \%)$ & $27(5 \%)$ & $37(0 \%)$ & $30(3 \%)$ & $43(0 \%)$ \\
\hline Other nervous disorders & $63(4 \%)$ & $233(1 \%)$ & $23(4 \%)$ & $92(1 \%)$ & $40(3 \%)$ & $141(1 \%)$ \\
\hline Eye disease & $311(18 \%)$ & $3163(14 \%)$ & $87(16 \%)$ & 1199 (12\%) & $224(19 \%)$ & $1964(16 \%)$ \\
\hline Ear disease & $61(4 \%)$ & $547(2 \%)$ & $10(2 \%)$ & $232(2 \%)$ & $51(4 \%)$ & 315 (3\%) \\
\hline Hypertension & $552(32 \%)$ & $6801(31 \%)$ & $148(27 \%)$ & $3036(31 \%)$ & $404(35 \%)$ & $3765(31 \%)$ \\
\hline Stroke & $256(15 \%)$ & $598(3 \%)$ & $136(25 \%)$ & $376(4 \%)$ & $120(10 \%)$ & $222(2 \%)$ \\
\hline Ischemic heart disease & $164(10 \%)$ & $1195(5 \%)$ & $60(11 \%)$ & $724(7 \%)$ & $104(9 \%)$ & $471(4 \%)$ \\
\hline Other circulatory diseases & $128(7 \%)$ & $957(4 \%)$ & $57(10 \%)$ & $485(5 \%)$ & $71(6 \%)$ & $472(4 \%)$ \\
\hline Cold & $9(1 \%)$ & 107 (0\%) & $4(1 \%)$ & $47(0 \%)$ & $5(0 \%)$ & $60(0 \%)$ \\
\hline Allergic rhinitis & $29(2 \%)$ & $528(2 \%)$ & $9(2 \%)$ & $234(2 \%)$ & $20(2 \%)$ & $294(2 \%)$ \\
\hline COPD & $19(1 \%)$ & $71(0 \%)$ & $13(2 \%)$ & $56(1 \%)$ & $6(1 \%)$ & $15(0 \%)$ \\
\hline Asthma & $47(3 \%)$ & $372(2 \%)$ & $18(3 \%)$ & $160(2 \%)$ & $29(2 \%)$ & $212(2 \%)$ \\
\hline Other respiratory diseases & $68(4 \%)$ & $399(2 \%)$ & $29(5 \%)$ & $224(2 \%)$ & $39(3 \%)$ & $175(1 \%)$ \\
\hline Stomach/duodenum disease & $63(4 \%)$ & $844(4 \%)$ & $20(4 \%)$ & $425(4 \%)$ & $43(4 \%)$ & $419(3 \%)$ \\
\hline Liver/gall bladder disease & $54(3 \%)$ & $471(2 \%)$ & $22(4 \%)$ & $252(3 \%)$ & $32(3 \%)$ & $219(2 \%)$ \\
\hline Other digestive diseases & $68(4 \%)$ & $485(2 \%)$ & $25(5 \%)$ & $243(2 \%)$ & $43(4 \%)$ & $242(2 \%)$ \\
\hline Dental diseases & $78(5 \%)$ & $1749(8 \%)$ & $25(5 \%)$ & $797(8 \%)$ & $53(5 \%)$ & $952(8 \%)$ \\
\hline Atopic dermatitis & $10(1 \%)$ & $88(0 \%)$ & $4(1 \%)$ & $50(1 \%)$ & $6(1 \%)$ & $38(0 \%)$ \\
\hline Other skin disease & $71(4 \%)$ & $543(2 \%)$ & $31(6 \%)$ & $321(3 \%)$ & $40(3 \%)$ & $222(2 \%)$ \\
\hline Gout & $19(1 \%)$ & $356(2 \%)$ & $13(2 \%)$ & $328(3 \%)$ & $6(1 \%)$ & $28(0 \%)$ \\
\hline Rheumatoid arthritis & $61(4 \%)$ & $313(1 \%)$ & $6(1 \%)$ & $73(1 \%)$ & $55(5 \%)$ & $240(2 \%)$ \\
\hline Arthropathy & $150(9 \%)$ & $1232(6 \%)$ & $22(4 \%)$ & $345(3 \%)$ & $128(11 \%)$ & $887(7 \%)$ \\
\hline Stiff shoulder & $88(5 \%)$ & $1256(6 \%)$ & $21(4 \%)$ & $361(4 \%)$ & $67(6 \%)$ & $895(7 \%)$ \\
\hline Low back pain & $282(16 \%)$ & $2639(12 \%)$ & $75(14 \%)$ & $990(10 \%)$ & $207(18 \%)$ & $1649(14 \%)$ \\
\hline Osteoporosis & $214(12 \%)$ & $1090(5 \%)$ & $17(3 \%)$ & $59(1 \%)$ & $197(17 \%)$ & $1031(8 \%)$ \\
\hline Kidney disease & $96(6 \%)$ & $438(2 \%)$ & $40(7 \%)$ & $266(3 \%)$ & $56(5 \%)$ & $172(1 \%)$ \\
\hline Prostatic hyperplasia & $76(4 \%)$ & $873(4 \%)$ & $76(14 \%)$ & $873(9 \%)$ & - & - \\
\hline $\begin{array}{l}\text { Menopause or postmenopausal } \\
\text { disorders }\end{array}$ & $4(0 \%)$ & $20(0 \%)$ & - & - & $4(0 \%)$ & $20(0 \%)$ \\
\hline Fracture & $93(5 \%)$ & $243(1 \%)$ & $19(3 \%)$ & $74(1 \%)$ & $74(6 \%)$ & $169(1 \%)$ \\
\hline Injury other than fracture/burn & $22(1 \%)$ & $146(1 \%)$ & $5(1 \%)$ & $55(1 \%)$ & $17(1 \%)$ & $91(1 \%)$ \\
\hline Anemia/blood disorder & $47(3 \%)$ & $213(1 \%)$ & $16(3 \%)$ & $90(1 \%)$ & $31(3 \%)$ & $123(1 \%)$ \\
\hline Cancer & $25(1 \%)$ & $339(2 \%)$ & $10(2 \%)$ & $185(2 \%)$ & $15(1 \%)$ & $154(1 \%)$ \\
\hline \multicolumn{7}{|l|}{ Have worries and stress } \\
\hline No & $518(30 \%)$ & $12,635(57 \%)$ & $153(28 \%)$ & $6072(62 \%)$ & $365(31 \%)$ & $6563(54 \%)$ \\
\hline Yes & $1151(67 \%)$ & $8972(41 \%)$ & $371(68 \%)$ & $3623(37 \%)$ & $780(67 \%)$ & $5349(44 \%)$ \\
\hline Missing & $49(3 \%)$ & $405(2 \%)$ & $24(4 \%)$ & $175(2 \%)$ & $25(2 \%)$ & $230(2 \%)$ \\
\hline $\begin{array}{l}\text { Consulting family about } \\
\text { worries and stress }\end{array}$ & $630(37 \%)$ & $3916(18 \%)$ & $178(32 \%)$ & $1432(15 \%)$ & 452 (39\%) & $2484(20 \%)$ \\
\hline $\begin{array}{l}\text { Consulting friends/ } \\
\text { acquaintances }\end{array}$ & $133(8 \%)$ & 1896 (9\%) & $23(4 \%)$ & $480(5 \%)$ & $110(9 \%)$ & $1416(12 \%)$ \\
\hline Consulting boss at work/ & $2(0 \%)$ & $22(0 \%)$ & $0(0 \%)$ & $12(0 \%)$ & $2(0 \%)$ & $10(0 \%)$ \\
\hline
\end{tabular}


Table 1 Basic characteristics of participants aged $\geq 65$ years with and without LTC certification (Continued)

\begin{tabular}{|c|c|c|c|c|c|c|}
\hline & \multicolumn{2}{|c|}{ Total $(n=23,730)$} & \multicolumn{2}{|c|}{ Men $(n=10,418)$} & \multicolumn{2}{|c|}{ Women $(n=13,312)$} \\
\hline & $\begin{array}{l}\text { Certified } \\
(n=1718)\end{array}$ & $\begin{array}{l}\text { Non-certified } \\
(n=22,012)\end{array}$ & $\begin{array}{l}\text { Certified } \\
(n=548)\end{array}$ & $\begin{array}{l}\text { Non-certified } \\
(n=9870)\end{array}$ & $\begin{array}{l}\text { Certified } \\
(n=1170)\end{array}$ & $\begin{array}{l}\text { Non- } \\
\text { certified } \\
(n=12,142)\end{array}$ \\
\hline \multicolumn{7}{|l|}{ teacher at school } \\
\hline Consulting public institutions & $142(8 \%)$ & $358(2 \%)$ & $53(10 \%)$ & $146(1 \%)$ & $89(8 \%)$ & $212(2 \%)$ \\
\hline Consulting doctors & $515(30 \%)$ & $2494(11 \%)$ & $182(33 \%)$ & 1109 (11\%) & $333(28 \%)$ & $1385(11 \%)$ \\
\hline Consulting others & $67(4 \%)$ & $368(2 \%)$ & $20(4 \%)$ & $162(2 \%)$ & $47(4 \%)$ & $206(2 \%)$ \\
\hline Cannot consult anyone & $35(2 \%)$ & $416(2 \%)$ & $12(2 \%)$ & $178(2 \%)$ & $23(2 \%)$ & $238(2 \%)$ \\
\hline Do not know where to consult & $27(2 \%)$ & $279(1 \%)$ & $9(2 \%)$ & $127(1 \%)$ & $18(2 \%)$ & $152(1 \%)$ \\
\hline No need to consult & $109(6 \%)$ & $1878(9 \%)$ & $45(8 \%)$ & $930(9 \%)$ & $64(5 \%)$ & $948(8 \%)$ \\
\hline \multicolumn{7}{|l|}{ K6 total score } \\
\hline$<13$ & $1302(76 \%)$ & 19,404 (88\%) & 402 (73\%) & 8835 (90\%) & 900 (77\%) & $10,569(87 \%)$ \\
\hline$\geq 13$ & $180(10 \%)$ & $521(2 \%)$ & $57(10 \%)$ & $197(2 \%)$ & $123(11 \%)$ & $324(3 \%)$ \\
\hline Missing & 236 (14\%) & 2087 (9\%) & 89 (16\%) & 838 (8\%) & 147 (13\%) & $1249(10 \%)$ \\
\hline
\end{tabular}

Data are presented as $\mathrm{N}(\%)$

Abbreviations: LTC long-term care, COPD chronic obstructive pulmonary disease

${ }^{a}$ The disposable income of a household divided by the square root of the number of people in the household

stronger effect in the women than men (Table 2), the interaction term between sex and age group was included in the multivariate analysis. All variables including sociodemographic status, subjective symptoms, diseases for which participants regularly visited the hospital, and worries and stress were entered simultaneously (Model 1). While no significant difference was observed between men and women in the 65-69 years' reference age group (aOR 0.79), the interaction term with sex was significant in the 85-89 years (aOR 2.49) age group.

The presence of a spouse (aOR 0.42) was negatively associated with LTC certification, while presence of children separately was positively associated with LTC certification (aOR 1.21). Of the subjective symptoms, difficulty in limb movement (aOR 2.07), diarrhoea (aOR 2.04), incontinence (aOR 1.61), wheezing (aOR 1.52), cough/phlegmatic (OR 1.48), swollen/heavy feet (aOR 1.42), numb limbs (aOR 1.35) and insufficient sleep (aOR 1.33) were positively associated with LTC certification, while ringing ears (aOR 0.72) and forgetfulness (aOR 0.79) were negatively associated with it. Among the diseases, dementia (aOR 14.62), stroke (aOR 6.90), Parkinson's disease (aOR 4.37), chronic obstructive pulmonary disease (COPD) (aOR 3.44), fracture (aOR 2.96), rheumatoid arthritis (aOR 2.66), other nervous disorders (aOR 2.57), kidney diseases (aOR 2.16), other respiratory diseases (aOR 1.62), diabetes (aOR 1.60) and osteoporosis (aOR 1.37) were positively associated with LTC certification. In contrast, hypertension (aOR 0.66), stomach/duodenum diseases (aOR 0.63), dental diseases (aOR 0.54), stiff shoulders (aOR 0.60) and eye diseases (aOR 0.78) showed a negative association. Regarding consultations about the participants' worries and stress, public institutions (aOR 3.36), family (aOR 1.66) and doctors (aOR 1.54) showed positive associations with LTC certification, while consultations with friends or acquaintances (aOR 0.69) demonstrated a negative association. A K6 total score $\geq 13$ was positively associated with LTC certification (aOR 1.76).

For Model 2, the number of subjective symptoms $(0-2$ or 3 or more symptoms) and the number of diseases (02 or 3 or more diseases) were entered as independent variables, instead of individual symptoms or diseases (Table 3). Having three or more subjective symptoms (aOR 1.31) and regular hospital visits for three or more diseases (aOR 1.47) were associated with LTC certification in Model 2.

Variable selection was performed on Model 1; the resulting Model 1A is shown in Table 3. ROC curves were drawn by adapting Model $1 \mathrm{~A}$ or Model 2 to the testing dataset (Fig. 2). The AUCs for Model 1A and 2 were 0.903 and 0.847 , respectively.

As a sensitivity analysis, complete case analysis was performed on 13,812 participants aged $\geq 65$ years with no missing data in the training dataset, including 985 certified participants (Supplementary Table 1). The results were largely similar; however, regular hospital visits for COPD was not associated with LTC in the complete case analysis (aOR 1.07, CI 0.35-3.24). This discrepancy may be explained by the fact that $63.2 \%$ of the certified participants with COPD had missing values and were thus excluded from the complete case analysis, while only $26.7 \%$ of the participants aged $\geq 65$ years required exclusion due to missing data.

The rate of certification was largely dependent on age. The basic characteristics of the participants aged 40-64, 
Table 2 Non-adjusted odds ratios of LTC certification in participants aged $\geq 65$ years

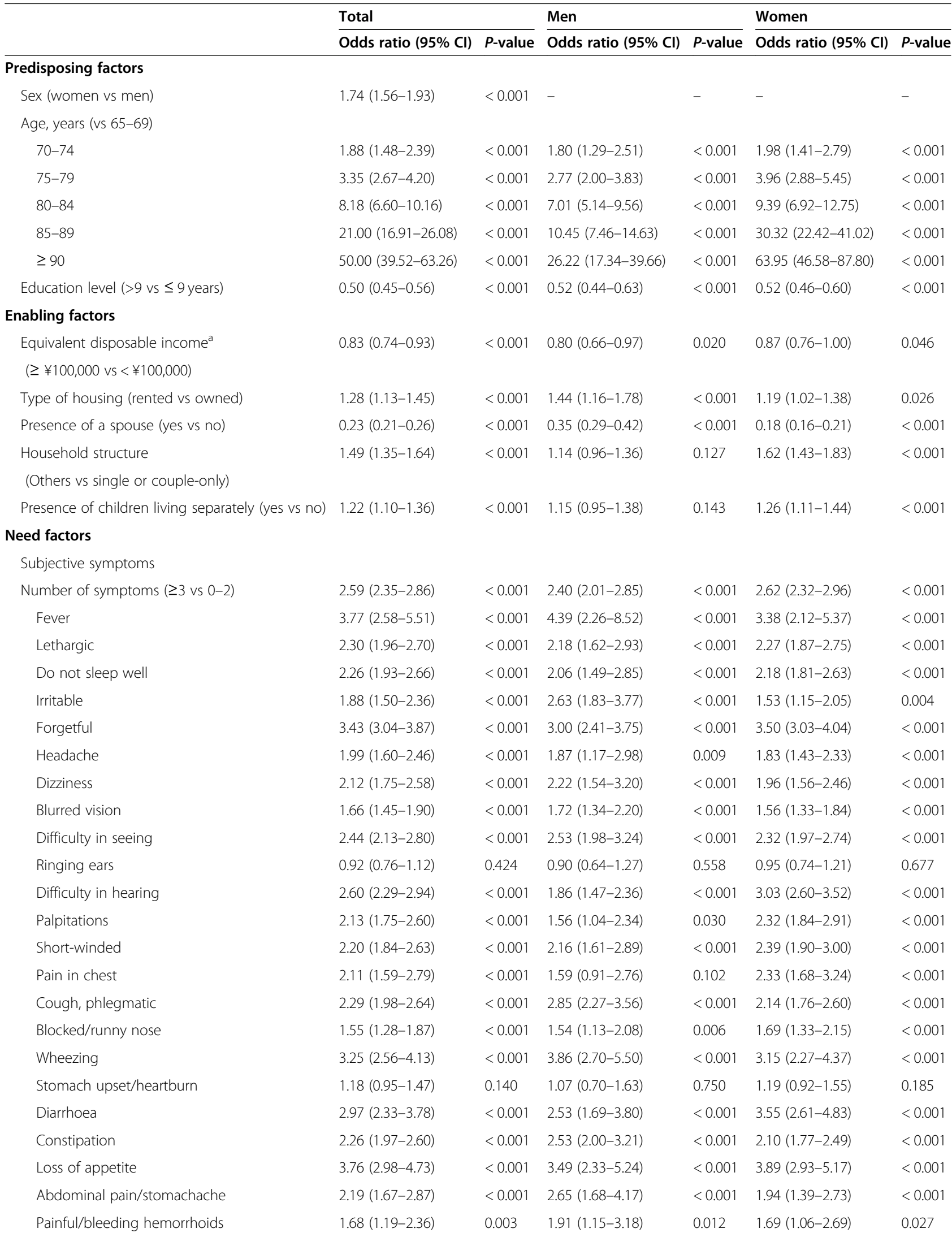


Table 2 Non-adjusted odds ratios of LTC certification in participants aged $\geq 65$ years (Continued)

\begin{tabular}{|c|c|c|c|c|c|c|}
\hline & \multicolumn{2}{|l|}{ Total } & \multicolumn{2}{|l|}{ Men } & \multicolumn{2}{|l|}{ Women } \\
\hline & Odds ratio $(95 \% \mathrm{Cl})$ & $P$-value & Odds ratio $(95 \% \mathrm{Cl})$ & $P$-value & Odds ratio $(95 \% \mathrm{Cl})$ & $P$-value \\
\hline Toothache & $1.22(0.92-1.62)$ & 0.175 & $1.29(0.80-2.06)$ & 0.298 & $1.20(0.84-1.72)$ & 0.308 \\
\hline Swollen/bleeding gums & $1.30(1.00-1.67)$ & 0.046 & $1.38(0.90-2.13)$ & 0.142 & $1.25(0.91-1.72)$ & 0.159 \\
\hline Difficulty in chewing & $2.32(1.97-2.72)$ & $<0.001$ & $2.22(1.67-2.96)$ & $<0.001$ & $2.33(1.92-2.83)$ & $<0.001$ \\
\hline Rash & $1.98(1.50-2.62)$ & $<0.001$ & $2.05(1.28-3.27)$ & 0.003 & $1.97(1.39-2.79)$ & $<0.001$ \\
\hline Itching & $1.93(1.65-2.27)$ & $<0.001$ & $1.97(1.53-2.55)$ & $<0.001$ & $2.11(1.71-2.60)$ & $<0.001$ \\
\hline Joint pain in hands/feet & $2.07(1.83-2.33)$ & $<0.001$ & $1.83(1.44-2.32)$ & $<0.001$ & $2.00(1.74-2.30)$ & $<0.001$ \\
\hline Difficulty in limb movement & $5.77(5.14-6.47)$ & $<0.001$ & $6.97(5.69-8.54)$ & $<0.001$ & $5.01(4.35-5.77)$ & $<0.001$ \\
\hline Numb limbs & $2.53(2.22-2.90)$ & $<0.001$ & $2.70(2.14-3.39)$ & $<0.001$ & $2.45(2.08-2.89)$ & $<0.001$ \\
\hline Cold limbs & $2.87(2.48-3.32)$ & $<0.001$ & $3.38(2.59-4.41)$ & $<0.001$ & $2.51(2.11-2.99)$ & $<0.001$ \\
\hline Swollen/heavy feet & $3.86(3.37-4.43)$ & $<0.001$ & $4.63(3.56-6.01)$ & $<0.001$ & $3.31(2.82-3.88)$ & $<0.001$ \\
\hline Difficulty in/painful urination & $2.12(1.67-2.69)$ & $<0.001$ & $2.10(1.51-2.91)$ & $<0.001$ & $3.38(2.34-4.86)$ & $<0.001$ \\
\hline Frequent urination & $2.04(1.76-2.36)$ & $<0.001$ & $1.73(1.36-2.20)$ & $<0.001$ & $2.65(2.19-3.19)$ & $<0.001$ \\
\hline Incontinence & $5.19(4.42-6.10)$ & $<0.001$ & $5.47(4.05-7.38)$ & $<0.001$ & $4.77(3.94-5.77)$ & $<0.001$ \\
\hline Injury including cut, burn & $2.02(1.26-3.24)$ & 0.004 & $1.21(0.44-3.33)$ & 0.718 & $2.47(1.43-4.27)$ & 0.001 \\
\hline \multicolumn{7}{|l|}{ Regular hospital visits } \\
\hline Number of diseases ( $\geq 3$ vs $0-2$ ) & $2.36(2.13-2.61)$ & $<0.001$ & $2.37(1.99-2.84)$ & $<0.001$ & $2.32(2.05-2.63)$ & $<0.001$ \\
\hline Diabetes & $1.46(1.27-1.68)$ & $<0.001$ & $1.70(1.37-2.11)$ & $<0.001$ & $1.47(1.22-1.78)$ & $<0.001$ \\
\hline Obesity & $1.14(0.73-1.79)$ & 0.559 & $1.44(0.66-3.13)$ & 0.355 & $0.98(0.57-1.71)$ & 0.950 \\
\hline Hyperlipidemia & $0.73(0.61-0.87)$ & $<0.001$ & $1.00(0.71-1.41)$ & 0.994 & $0.58(0.47-0.72)$ & $<0.001$ \\
\hline Thyroid disease & $1.27(0.92-1.76)$ & 0.143 & $2.96(1.56-5.63)$ & $<0.001$ & $0.89(0.61-1.31)$ & 0.564 \\
\hline Mental illness & $2.62(1.95-3.51)$ & $<0.001$ & $2.40(1.23-4.67)$ & 0.010 & $2.40(1.73-3.34)$ & $<0.001$ \\
\hline Dementia & $28.41(23.42-34.47)$ & $<0.001$ & $26.46(19.23-36.42)$ & $<0.001$ & $28.97(22.64-37.07)$ & $<0.001$ \\
\hline Parkinson's disease & $9.38(6.66-13.23)$ & $<0.001$ & $13.81(8.34-22.86)$ & $<0.001$ & $7.36(4.60-11.78)$ & $<0.001$ \\
\hline Other nervous disorders & $3.55(2.67-4.71)$ & $<0.001$ & $4.67(2.93-7.43)$ & $<0.001$ & $3.00(2.10-4.28)$ & $<0.001$ \\
\hline Eye disease & $1.31(1.15-1.49)$ & $<0.001$ & $1.37(1.08-1.74)$ & 0.009 & $1.22(1.05-1.42)$ & 0.011 \\
\hline Ear disease & $1.44(1.10-1.89)$ & 0.008 & $0.77(0.41-1.47)$ & 0.432 & $1.70(1.26-2.30)$ & $<0.001$ \\
\hline Hypertension & $1.05(0.95-1.17)$ & 0.323 & $0.83(0.69-1.01)$ & 0.068 & $1.16(1.03-1.32)$ & 0.019 \\
\hline Stroke & $6.26(5.36-7.31)$ & $<0.001$ & $8.38(6.73-10.44)$ & $<0.001$ & $6.10(4.85-7.69)$ & $<0.001$ \\
\hline Ischemic heart disease & $1.83(1.54-2.18)$ & $<0.001$ & $1.56(1.18-2.06)$ & 0.002 & $2.40(1.93-3.00)$ & $<0.001$ \\
\hline Other circulatory diseases & $1.77(1.46-2.14)$ & $<0.001$ & $2.25(1.69-3.01)$ & $<0.001$ & $1.59(1.23-2.05)$ & $<0.001$ \\
\hline Cold & $1.07(0.54-2.13)$ & 0.835 & $1.54(0.55-4.29)$ & 0.409 & $0.86(0.34-2.14)$ & 0.745 \\
\hline Allergic rhinitis & $0.70(0.48-1.02)$ & 0.060 & $0.69(0.35-1.35)$ & 0.277 & $0.70(0.44-1.10)$ & 0.121 \\
\hline COPD & $3.45(2.07-5.73)$ & $<0.001$ & $4.27(2.32-7.85)$ & $<0.001$ & $4.14(1.60-10.70)$ & 0.003 \\
\hline Asthma & $1.63(1.20-2.22)$ & 0.002 & $2.07(1.26-3.39)$ & 0.004 & $1.42(0.96-2.11)$ & 0.079 \\
\hline Other respiratory diseases & $2.23(1.71-2.89)$ & $<0.001$ & $2.41(1.62-3.59)$ & $<0.001$ & $2.34(1.65-3.34)$ & $<0.001$ \\
\hline Stomach/duodenum disease & $0.95(0.73-1.24)$ & 0.711 & $0.84(0.53-1.33)$ & 0.466 & $1.06(0.77-1.46)$ & 0.715 \\
\hline Liver/gall bladder disease & $1.48(1.11-1.97)$ & 0.007 & $1.60(1.03-2.50)$ & 0.038 & $1.52(1.05-2.22)$ & 0.028 \\
\hline Other digestive diseases & $1.82(1.41-2.36)$ & $<0.001$ & $1.90(1.25-2.89)$ & 0.003 & $1.87(1.34-2.60)$ & $<0.001$ \\
\hline Dental diseases & $0.55(0.44-0.69)$ & $<0.001$ & $0.55(0.36-0.82)$ & 0.004 & $0.55(0.42-0.74)$ & $<0.001$ \\
\hline Atopic dermatitis & $1.45(0.75-2.80)$ & 0.263 & $1.45(0.52-4.02)$ & 0.478 & $1.63(0.69-3.87)$ & 0.266 \\
\hline Other skin disease & $1.70(1.32-2.19)$ & $<0.001$ & $1.79(1.22-2.61)$ & 0.003 & $1.89(1.34-2.66)$ & $<0.001$ \\
\hline Gout & $0.68(0.43-1.08)$ & 0.101 & $0.71(0.40-1.24)$ & 0.228 & $2.22(0.92-5.37)$ & 0.077 \\
\hline Rheumatoid arthritis & $2.55(1.93-3.36)$ & $<0.001$ & $1.49(0.64-3.44)$ & 0.351 & $2.43(1.80-3.28)$ & $<0.001$ \\
\hline
\end{tabular}


Table 2 Non-adjusted odds ratios of LTC certification in participants aged $\geq 65$ years (Continued)

\begin{tabular}{|c|c|c|c|c|c|c|}
\hline & \multicolumn{2}{|l|}{ Total } & \multicolumn{2}{|l|}{ Men } & \multicolumn{2}{|l|}{ Women } \\
\hline & Odds ratio $(95 \% \mathrm{Cl})$ & $P$-value & Odds ratio $(95 \% \mathrm{Cl})$ & $P$-value & Odds ratio $(95 \% \mathrm{Cl})$ & $P$-value \\
\hline Arthropathy & $1.61(1.35-1.92)$ & $<0.001$ & $1.16(0.75-1.80)$ & 0.515 & $1.55(1.27-1.89)$ & $<0.001$ \\
\hline Stiff shoulder & $0.89(0.71-1.11)$ & 0.301 & $1.05(0.67-1.65)$ & 0.825 & $0.76(0.59-0.98)$ & 0.034 \\
\hline Low back pain & $1.44(1.26-1.64)$ & $<0.001$ & $1.43(1.11-1.84)$ & 0.006 & $1.36(1.16-1.59)$ & $<0.001$ \\
\hline Osteoporosis & $2.72(2.33-3.18)$ & $<0.001$ & $5.34(3.09-9.22)$ & $<0.001$ & $2.17(1.84-2.56)$ & $<0.001$ \\
\hline Kidney disease & $2.91(2.32-3.65)$ & $<0.001$ & $2.85(2.02-4.02)$ & $<0.001$ & $3.48(2.56-4.73)$ & $<0.001$ \\
\hline Prostatic hyperplasia & $1.12(0.88-1.42)$ & 0.364 & $1.66(1.29-2.14)$ & $<0.001$ & - & - \\
\hline Menopause or postmenopausal disorders & $2.56(0.87-7.49)$ & 0.087 & - & - & $2.07(0.71-6.06)$ & 0.185 \\
\hline Fracture & $5.11(4.01-6.53)$ & $<0.001$ & $4.77(2.86-7.95)$ & $<0.001$ & $4.76(3.60-6.30)$ & $<0.001$ \\
\hline Injury other than fracture/burn & $1.94(1.23-3.04)$ & 0.004 & $1.65(0.66-4.13)$ & 0.288 & $1.94(1.15-3.27)$ & 0.013 \\
\hline Anemia/blood disorder & $2.87(2.08-3.95)$ & $<0.001$ & $3.28(1.91-5.62)$ & $<0.001$ & $2.64(1.78-3.94)$ & $<0.001$ \\
\hline Cancer & $0.94(0.63-1.42)$ & 0.772 & $0.98(0.51-1.85)$ & 0.939 & $1.01(0.59-1.71)$ & 0.985 \\
\hline \multicolumn{7}{|l|}{ Consult about worries and stress with (yes vs no) } \\
\hline Family & $2.74(2.47-3.04)$ & $<0.001$ & $2.97(2.46-3.59)$ & $<0.001$ & $2.48(2.18-2.81)$ & $<0.001$ \\
\hline Friends/acquaintances & $0.90(0.75-1.08)$ & 0.261 & $0.88(0.57-1.35)$ & 0.563 & $0.79(0.64-0.97)$ & 0.022 \\
\hline Boss at work/teacher at school & $1.18(0.28-5.01)$ & 0.825 & 0.00 (0.00- inf) & 0.964 & $2.08(0.46-9.52)$ & 0.344 \\
\hline Public institutions & $5.52(4.51-6.75)$ & $<0.001$ & $7.36(5.30-10.21)$ & $<0.001$ & $4.65(3.60-6.01)$ & $<0.001$ \\
\hline Doctors & $3.42(3.06-3.83)$ & $<0.001$ & $4.12(3.41-4.98)$ & $<0.001$ & $3.12(2.71-3.58)$ & $<0.001$ \\
\hline Other than above & $2.41(1.85-3.15)$ & $<0.001$ & $2.34(1.45-3.75)$ & $<0.001$ & $2.43(1.76-3.36)$ & $<0.001$ \\
\hline Cannot consult anyone & $1.09(0.77-1.55)$ & 0.624 & $1.25(0.69-2.26)$ & 0.455 & $1.01(0.65-1.55)$ & 0.980 \\
\hline Do not know where to consult & $1.26(0.84-1.87)$ & 0.260 & $1.32(0.67-2.60)$ & 0.429 & $1.24(0.76-2.02)$ & 0.400 \\
\hline No need to consult & $0.73(0.60-0.90)$ & 0.002 & $0.89(0.65-1.21)$ & 0.446 & $0.68(0.53-0.89)$ & 0.004 \\
\hline K6 total score ( $\geq 13$ vs < 13) & $5.15(4.31-6.16)$ & $<0.001$ & $6.36(4.66-8.68)$ & $<0.001$ & $4.46(3.58-5.55)$ & $<0.001$ \\
\hline
\end{tabular}

Abbreviations: LTC long-term care, Cl confidence interval, COPD chronic obstructive pulmonary disease

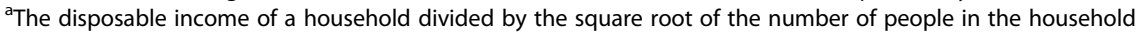

65-74 and $\geq 75$ years with or without LTC certification are shown in Supplementary Table 2. The results of the univariate analyses are shown in Supplementary Table 3. While the overall tendency was similar across the age groups, the diseases showed higher ORs in the participants aged 40-64 years than in the older age groups.

Certified participants aged $\geq 65$ years with 'a lower degree of independence' were determined as explained in the Methods section. Of the 1718 certified participants aged $\geq 65$ years, 430 were classified as having 'a lower degree of independence'. The difference between certified participants with 'a lower degree of independence' and those with 'a higher degree of independence' was evaluated in univariate analysis (Supplementary Table 4). In the univariate analysis among certified participants, while dementia, Parkinson's disease and stroke were associated with a 'lower degree of independence', fracture, COPD, kidney disease, osteoporosis, rheumatoid arthritis and diabetes were not (Supplementary Table 4). Multivariate analyses were performed to determine adjusted odds ratios of LTC certification with a lower or higher degree of independence, using the same set of variables used in the aforementioned Model 2 (Supplementary Table 5). 'Neither single nor couple-only household' was associated with a lower degree of independence; however, 'the absence of a spouse' was not associated with a lower degree of independence.

Although the eligibility for LTC certification in Japan is determined based on ADL, other factors might play a role in decision-making to apply for one in people with impaired ADL. To address this point, the differences between certified and non-certified participants among participants who answered 'I need assistance or supervision due to disabilities or impaired physical function' were evaluated (Supplementary Table 6), based on the assumption that most of the participants without LTC certification have not applied for one. Factors associated with LTC certification included female sex, older age group, household structure (neither single nor coupleonly), fever, difficulty in limb movement, numb limbs, swollen/heavy feet, incontinence, dementia, Parkinson's disease, stroke, other skin diseases, osteoporosis, 
Table 3 Adjusted odds ratios of factors associated with LTC certification before and after variable selection

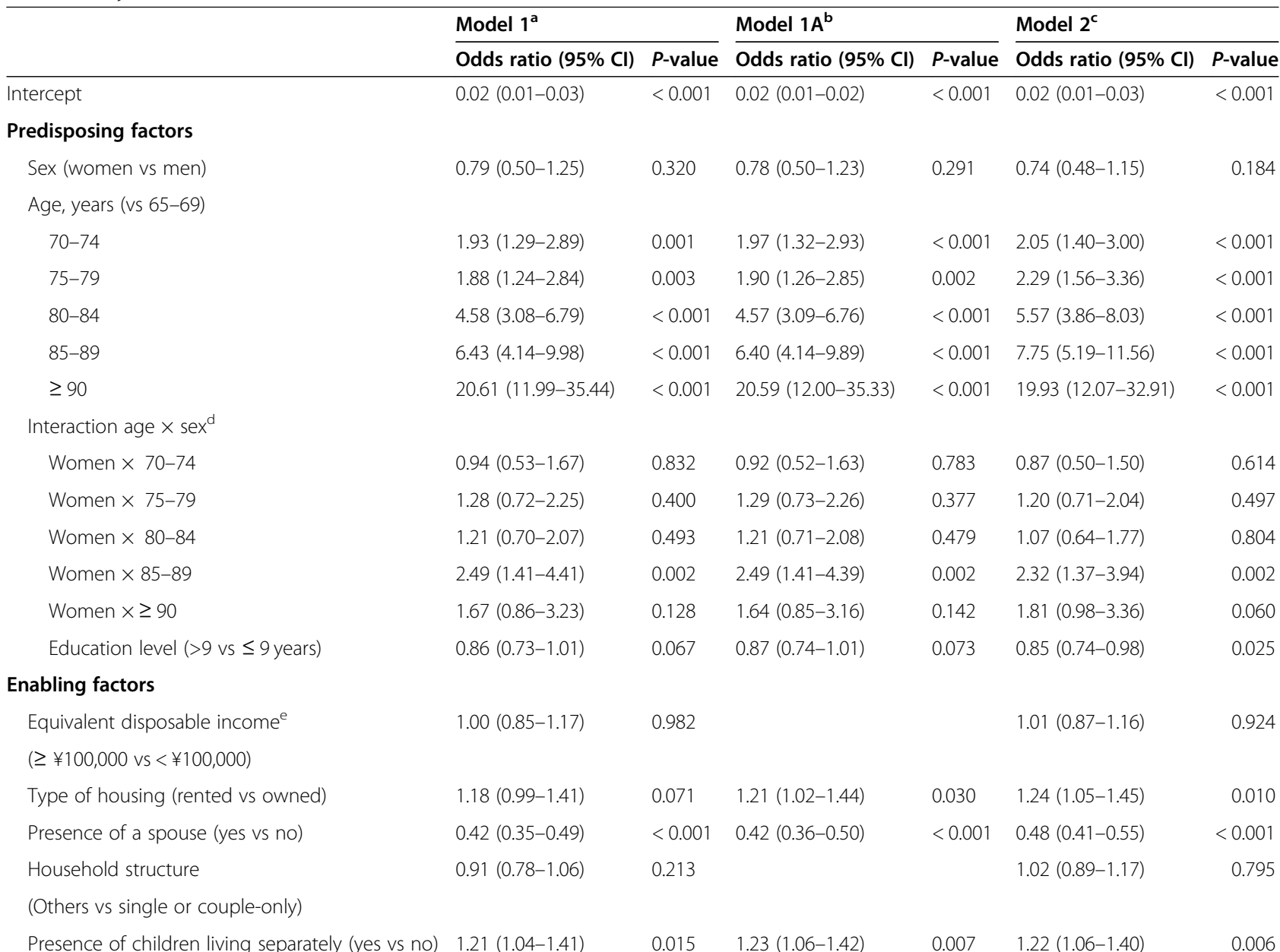

\section{Need factors}

Subjective symptoms

Number of symptoms ( $\geq 3$ vs $0-2$ )

Fever
Lethargic
Do not sleep well
Irritable
Forgetful
Headache
Dizziness
Blurred vision
Difficulty in seeing
Ringing ears
Difficulty in hearing
Palpitations
Short-winded
Pain in chest
Cough, phlegmatic
Blocked/runny nose

$\begin{array}{llll}1.15(0.60-2.21) & 0.665 & & \\ 0.84(0.63-1.11) & 0.208 & & \\ 1.33(1.02-1.74) & 0.038 & 1.35(1.05-1.75) & 0.021 \\ 0.97(0.66-1.41) & 0.854 & & \\ 0.79(0.63-0.98) & 0.036 & 0.80(0.65-0.99) & 0.043 \\ 1.12(0.78-1.61) & 0.529 & & \\ 1.05(0.77-1.45) & 0.745 & & \\ 0.87(0.70-1.10) & 0.250 & & \\ 1.15(0.90-1.46) & 0.257 & & 0.022 \\ 0.72(0.53-0.97) & 0.029 & 0.71(0.53-0.95) & 0.152 \\ 0.85(0.68-1.06) & 0.147 & 0.86(0.69-1.06) & 0.139 \\ 0.78(0.56-1.10) & 0.163 & 0.78(0.56-1.08) & 0.109 \\ 0.75(0.54-1.04) & 0.084 & 0.77(0.56-1.06) & \\ 0.78(0.49-1.24) & 0.293 & & 0.002 \\ 1.48(1.15-1.89) & 0.002 & 1.46(1.15-1.86) & 0.165 \\ 0.82(0.60-1.12) & 0.215 & 0.81(0.60-1.09) & \end{array}$


Table 3 Adjusted odds ratios of factors associated with LTC certification before and after variable selection (Continued)

\begin{tabular}{|c|c|c|c|c|c|c|}
\hline & \multicolumn{2}{|l|}{ Model $1^{\mathrm{a}}$} & \multicolumn{2}{|l|}{ Model $1 A^{b}$} & \multicolumn{2}{|l|}{ Model $2^{c}$} \\
\hline & Odds ratio $(95 \% \mathrm{Cl})$ & $P$-value & Odds ratio $(95 \% \mathrm{Cl})$ & $P$-value & Odds ratio $(95 \% \mathrm{Cl})$ & $P$-value \\
\hline Wheezing & $1.52(1.00-2.28)$ & 0.048 & $1.48(1.00-2.18)$ & 0.051 & & \\
\hline Stomach upset/heartburn & $0.73(0.51-1.05)$ & 0.094 & $0.74(0.52-1.04)$ & 0.086 & & \\
\hline Diarrhoea & $2.04(1.38-3.00)$ & $<0.001$ & $2.15(1.48-3.13)$ & $<0.001$ & & \\
\hline Constipation & $0.90(0.72-1.14)$ & 0.392 & & & & \\
\hline Loss of appetite & $1.27(0.87-1.85)$ & 0.223 & & & & \\
\hline Abdominal pain/stomachache & $1.13(0.72-1.77)$ & 0.594 & & & & \\
\hline Painful/bleeding hemorrhoids & $1.29(0.78-2.13)$ & 0.326 & & & & \\
\hline Toothache & $0.82(0.53-1.26)$ & 0.364 & & & & \\
\hline Swollen/bleeding gums & $1.14(0.77-1.67)$ & 0.517 & & & & \\
\hline Difficulty in chewing & $1.18(0.90-1.54)$ & 0.238 & & & & \\
\hline Rash & $0.87(0.54-1.40)$ & 0.558 & & & & \\
\hline Itching & $1.05(0.80-1.39)$ & 0.705 & & & & \\
\hline Joint pain in hands/feet & $0.90(0.73-1.11)$ & 0.313 & & & & \\
\hline Difficulty in limb movement & $2.07(1.70-2.53)$ & $<0.001$ & $2.08(1.72-2.51)$ & $<0.001$ & & \\
\hline Numb limbs & $1.35(1.08-1.69)$ & 0.008 & $1.34(1.08-1.67)$ & 0.007 & & \\
\hline Cold limbs & $1.04(0.80-1.34)$ & 0.783 & & & & \\
\hline Swollen/heavy feet & $1.42(1.12-1.80)$ & 0.004 & $1.43(1.13-1.79)$ & 0.002 & & \\
\hline Difficulty in/painful urination & $1.00(0.68-1.48)$ & 0.988 & & & & \\
\hline Frequent urination & $1.12(0.89-1.42)$ & 0.336 & & & & \\
\hline Incontinence & $1.61(1.23-2.11)$ & $<0.001$ & $1.61(1.24-2.09)$ & $<0.001$ & & \\
\hline Injury including cut, burn & $0.60(0.28-1.29)$ & 0.195 & & & & \\
\hline \multicolumn{7}{|l|}{ Regular hospital visits (yes vs no) } \\
\hline Number of diseases ( $\geq 3$ vs $0-2$ ) & & & & & $1.47(1.28-1.69)$ & $<0.001$ \\
\hline Diabetes & $1.60(1.31-1.96)$ & $<0.001$ & $1.60(1.32-1.96)$ & $<0.001$ & & \\
\hline Obesity & $0.65(0.30-1.40)$ & 0.270 & $0.60(0.28-1.28)$ & 0.187 & & \\
\hline Hyperlipidemia & $0.95(0.73-1.23)$ & 0.686 & & & & \\
\hline Thyroid disease & $0.94(0.58-1.51)$ & 0.794 & & & & \\
\hline Mental illness & $1.42(0.90-2.24)$ & 0.132 & $1.40(0.90-2.20)$ & 0.138 & & \\
\hline Dementia & $14.62(11.05-19.35)$ & $<0.001$ & $14.34(10.87-18.93)$ & $<0.001$ & & \\
\hline Parkinson's disease & $4.37(2.54-7.51)$ & $<0.001$ & $4.15(2.42-7.11)$ & $<0.001$ & & \\
\hline Other nervous disorders & $2.57(1.69-3.89)$ & $<0.001$ & $2.49(1.65-3.76)$ & $<0.001$ & & \\
\hline Eye disease & $0.78(0.64-0.95)$ & 0.016 & $0.78(0.64-0.94)$ & 0.010 & & \\
\hline Ear disease & $0.75(0.49-1.14)$ & 0.181 & $0.75(0.49-1.14)$ & 0.176 & & \\
\hline Hypertension & $0.66(0.57-0.77)$ & $<0.001$ & $0.66(0.57-0.77)$ & $<0.001$ & & \\
\hline Stroke & $6.90(5.48-8.67)$ & $<0.001$ & $6.90(5.49-8.65)$ & $<0.001$ & & \\
\hline Ischemic heart disease & $1.12(0.86-1.45)$ & 0.392 & & & & \\
\hline Other circulatory diseases & $0.90(0.67-1.20)$ & 0.466 & & & & \\
\hline Cold & $0.59(0.21-1.63)$ & 0.305 & & & & \\
\hline Allergic rhinitis & $0.77(0.45-1.32)$ & 0.334 & & & & \\
\hline COPD & $3.44(1.65-7.18)$ & 0.001 & $3.51(1.69-7.30)$ & $<0.001$ & & \\
\hline Asthma & $1.04(0.64-1.71)$ & 0.865 & & & & \\
\hline Other respiratory diseases & $1.62(1.11-2.38)$ & 0.013 & $1.55(1.06-2.26)$ & 0.022 & & \\
\hline Stomach/duodenum disease & $0.63(0.43-0.93)$ & 0.020 & $0.67(0.46-0.98)$ & 0.040 & & \\
\hline
\end{tabular}


Table 3 Adjusted odds ratios of factors associated with LTC certification before and after variable selection (Continued)

\begin{tabular}{|c|c|c|c|c|c|c|}
\hline & \multicolumn{2}{|l|}{ Model $1^{a}$} & \multicolumn{2}{|l|}{ Model $1 A^{b}$} & \multicolumn{2}{|l|}{ Model $2^{c}$} \\
\hline & Odds ratio $(95 \% \mathrm{Cl})$ & $P$-value & Odds ratio $(95 \% \mathrm{Cl})$ & $P$-value & Odds ratio $(95 \% \mathrm{Cl})$ & $P$-value \\
\hline Liver/gall bladder disease & $1.34(0.89-2.01)$ & 0.167 & & & & \\
\hline Other digestive diseases & $1.08(0.73-1.62)$ & 0.691 & & & & \\
\hline Dental diseases & $0.54(0.38-0.76)$ & $<0.001$ & $0.55(0.40-0.76)$ & $<0.001$ & & \\
\hline Atopic dermatitis & $1.51(0.60-3.80)$ & 0.381 & & & & \\
\hline Other skin disease & $1.40(0.93-2.11)$ & 0.104 & $1.43(0.98-2.09)$ & 0.063 & & \\
\hline Gout & $0.71(0.35-1.44)$ & 0.347 & & & & \\
\hline Rheumatoid arthritis & $2.66(1.78-3.98)$ & $<0.001$ & $2.58(1.74-3.81)$ & $<0.001$ & & \\
\hline Arthropathy & $1.05(0.80-1.38)$ & 0.711 & & & & \\
\hline Stiff shoulder & $0.60(0.43-0.83)$ & 0.002 & $0.61(0.45-0.83)$ & 0.001 & & \\
\hline Low back pain & $1.02(0.83-1.26)$ & 0.851 & & & & \\
\hline Osteoporosis & $1.37(1.08-1.75)$ & 0.011 & $1.39(1.09-1.76)$ & 0.007 & & \\
\hline Kidney disease & $2.16(1.53-3.06)$ & $<0.001$ & $2.22(1.59-3.11)$ & $<0.001$ & & \\
\hline Prostatic hyperplasia & $1.26(0.90-1.78)$ & 0.182 & $1.31(0.94-1.83)$ & 0.108 & & \\
\hline Menopause or postmenopausal disorders & $2.04(0.25-16.40)$ & 0.501 & & & & \\
\hline Fracture & $2.96(2.03-4.29)$ & $<0.001$ & $2.85(1.97-4.13)$ & $<0.001$ & & \\
\hline Injury other than fracture/burn & $1.35(0.69-2.61)$ & 0.378 & & & & \\
\hline Anemia/blood disorder & $1.34(0.82-2.19)$ & 0.236 & & & & \\
\hline Cancer & $0.94(0.54-1.63)$ & 0.828 & & & & \\
\hline \multicolumn{7}{|l|}{ Consult about worries and stress with (yes vs no) } \\
\hline Family & $1.66(1.40-1.97)$ & $<0.001$ & $1.65(1.40-1.95)$ & $<0.001$ & $1.65(1.42-1.93)$ & $<0.001$ \\
\hline Friends/acquaintances & $0.69(0.53-0.90)$ & 0.006 & $0.69(0.53-0.90)$ & 0.005 & $0.66(0.52-0.84)$ & $<0.001$ \\
\hline Boss at work/teacher at school & $2.47(0.29-21.17)$ & 0.409 & & & $1.81(0.29-11.32)$ & 0.525 \\
\hline Public institutions & $3.36(2.43-4.64)$ & $<0.001$ & $3.31(2.40-4.55)$ & $<0.001$ & $3.70(2.79-4.89)$ & $<0.001$ \\
\hline Doctors & $1.54(1.28-1.85)$ & $<0.001$ & $1.52(1.27-1.82)$ & $<0.001$ & $1.94(1.65-2.28)$ & $<0.001$ \\
\hline Other than above & $1.38(0.92-2.05)$ & 0.115 & $1.41(0.95-2.08)$ & 0.085 & $1.57(1.10-2.22)$ & 0.012 \\
\hline Cannot consult anyone & $1.26(0.75-2.11)$ & 0.382 & & & $1.35(0.85-2.12)$ & 0.201 \\
\hline Do not know where to consult & $1.26(0.68-2.34)$ & 0.460 & & & $1.21(0.70-2.08)$ & 0.496 \\
\hline No need to consult & $0.99(0.75-1.31)$ & 0.959 & & & $1.01(0.78-1.31)$ & 0.938 \\
\hline K6 total score ( $\geq 13$ vs < 13) & $1.76(1.32-2.36)$ & $<0.001$ & $1.83(1.38-2.43)$ & $<0.001$ & $2.56(2.01-3.26)$ & $<0.001$ \\
\hline
\end{tabular}

Abbreviations: LTC long-term care, Cl confidence interval, COPD chronic obstructive pulmonary disease

${ }^{\text {a }}$ Model 1 before variable selection

${ }^{\mathrm{b}}$ Model1A after variable selection

${ }^{\mathrm{c}}$ Model2 Subjective symptoms and regular hospital visits were clustered into $\geq 3$ or $0-2$ symptoms/diseases

${ }^{\mathrm{d}}$ Interaction term between sex and age groups

'The disposable income of a household divided by the square root of the number of people in the household

fracture, anaemia/blood disorder and lower degrees of independence in daily life activities.

\section{Discussion}

The present study investigated the factors associated with LTC certification using nationally representative data in Japan. We demonstrated that various factors including social, physical and psychological factors are associated with LTC certification, with the multivariate model showing good discrimination (AUC 0.903 and 0.847) (Fig. 2).
Regular hospital visits for dementia (aOR 14.62), stroke (aOR 6.90), Parkinson's disease (aOR 4.37), COPD (aOR 3.44), fracture (aOR 2.96), rheumatoid arthritis (aOR 2.66), kidney diseases (aOR 2.16), diabetes (aOR 1.60) and osteoporosis (aOR 1.37), difficulty in limb movement (aOR 2.07) and incontinence (aOR 1.61), were among those significantly associated with LTC certification in the multivariate analyses (Table 3), consistent with previous studies $[7,9,14,16]$. Regular hospital visits for COPD showed strikingly high ORs (aOR 3.44), even though 'respiratory diseases' including 

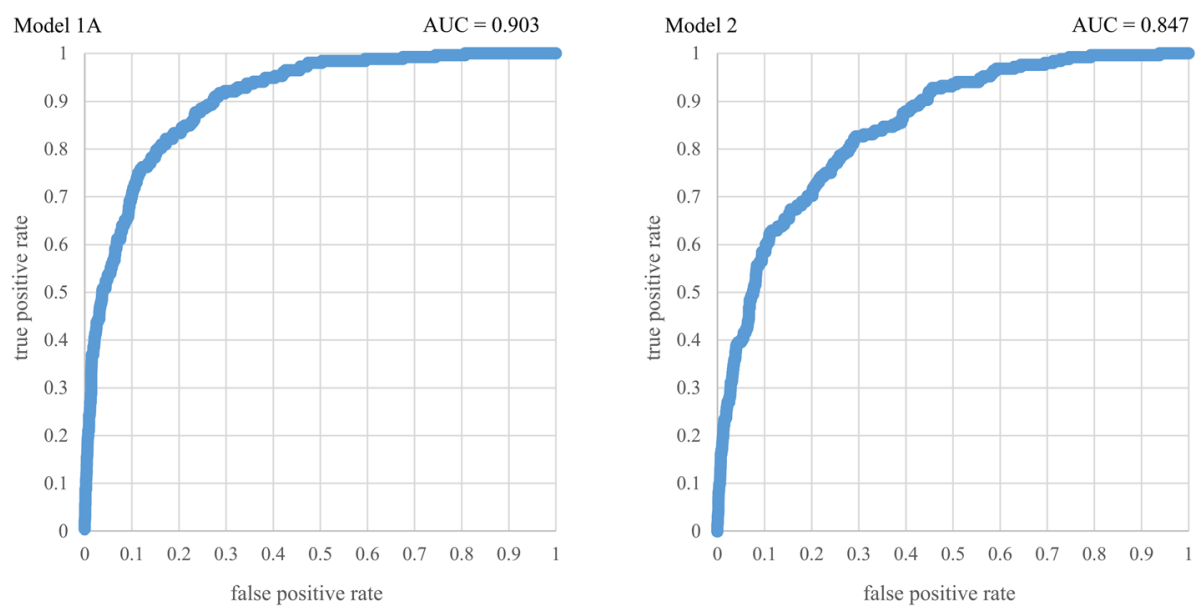

Fig. 2 Receiver operating characteristic curve for Model 1A and Model 2, a multivariate model explaining long-term care certification. The AUC for these models were 0.903 and 0.847 , respectively. AUC, area under the curve

COPD among others ranked only 10th as a cause of LTC in 2013 [10].

We did not detect an association between cancer and LTC (aOR 0.94, 95\% CI 0.54-1.63, Table 3) unlike previous studies $[7,9,22]$. As patients with ADL deteriorations due to cancer progression may survive and receive LTC for a relatively short time, their data may not have been captured owing to the cross-sectional survey design.

Regular hospital visits for hypertension was not associated with LTC certification in the univariate analysis (OR 1.05, 95\% CI 0.95-1.17, Table 2); however, it showed a negative association (aOR 0.66, 95\% CI 0.570.77 , Table 3 ) in the multivariate analysis. This is in contrast with previous studies which reported that hypertension is not associated with LTC certification [7, 16, 17, 22]. While most previous studies used the 'presence of hypertension' as a variable, 'regular visits to clinics or hospitals for hypertension' was used in this study. Therefore, our result indicates that the risk of LTC certification may reduce if hypertension is treated. Of note, only $31 \%$ of the participants aged $\geq 65$ years in this study reported that they regularly visited hospital for hypertension (Table 1), although more than $60 \%$ of the population aged $\geq 65$ years were estimated to have hypertension according to the 2013 National Health and Nutrition Survey [23]. In addition, as these participants were aware that they had hypertension and were willing to get treated, they are likely to have a high health literacy level, which could partly explain the negative association with LTC certification. In addition to hypertension, stiff shoulders (aOR 0.60), stomach/duodenum diseases (aOR 0.63), dental diseases (aOR 0.54) and eye diseases (aOR 0.78) were negatively associated with LTC certification. This may be because people who need LTC care are less likely to visit medical institutions for relatively mild diseases, as they prioritise treatment of more severe conditions. Alternatively, it is possible that some certified participants with multiple diseases underreported relatively mild diseases.

Among subjective symptoms, 'swollen/heavy feet' was significantly associated with LTC certification (Table 3), independently of 'difficulty in limb movement' and 'numb limbs'. Although these factors apparently have some overlaps, it can be speculated that some participants with swollen/heavy feet due to diseases such as heart diseases, kidney diseases, liver diseases, or varicose veins were free of musculoskeletal problems. Although 'forgetfulness' was positively associated with LTC certification in univariate analyses (Table 2), it was negatively associated in multivariate analyses (Table 3 ). This may be partly because people with dementia often underreport their symptoms.

As for psychological factors, severe psychological distress, as indicated by $\mathrm{K} 6$ scores $\geq 13$ (aOR 1.76), was associated with LTC (Table 3). Depression is thought to increase the risk of disability or frailty in older adults, which is at least partly explained by social inactivity [11, $24,25]$. Similarly, low social interaction levels were reported to be significant predictors of LTC certification [17] or functional decline [26] in older adults. Interestingly, our results show that consulting with friends or acquaintances about worries and stress was negatively associated with LTC (aOR 0.69); consultations at public institutions (aOR 3.36) or with family (aOR 1.66) or doctors (aOR 1.54) showed positive associations (Table 3). Having friends to talk to about worries and stress may indicate high social interaction levels, which could lower the risk of frailty. Intervention for mental health and the promotion of social interaction for the avoidance of isolation may be effective in preventing LTC in older adults. 
Concerning social factors, older age and the absence of a spouse were associated with LTC certification, consistent with previous reports [14-17]. Previous reports on the association between sex and LTC are inconsistent, with some showing no association $[14,16]$ and others demonstrating a low risk $[7,15]$ or high risk [22] in women. Our results suggest that the association between sex and LTC is largely dependent on age group, with no significant differences in the 65-69 years age group, but women were more likely to be certified at an older age (Table 3). These inconsistencies may be attributed to different compositions of age group and sex in each cohort.

Regarding education history, the findings have been mixed so far, with some suggesting that people with higher education levels are less likely to be caredependent [14] while others reported no association [16, $17,27]$. In our study, education for $>9$ years showed a tendency of negative association (aOR 0.86, 95\% CI $0.73-1.01$ ) in the multivariate analysis (Table 3).

In terms of enabling factors in the Andersen model, 'the absence of a spouse' and 'presence of children living separately' were associated with LTC certification (Table 3). 'Neither single nor couple-only household' was not associated with overall LTC certification (Table 3); however, it was associated with a lower degree of independence (Supplementary Table 5). Living with someone other than a spouse (e.g., children) did not affect LTC certification, which may be partly because the availability of family caregiving is not considered when determining the eligibility for LTC certification in Japan as described above [2]. The current study focused on people who were not in care facilities, and those with a lower degree of independence are more likely to be in care facilities, especially when family caregiving is unavailable. Living in a rented house was associated with LTC certification in the multivariate Models 1A and 2, but equivalent disposable income did not exhibit this association (Table 3 ).

The major strength of this study is the use of largescale nationally representative data for the identification of the factors associated with LTC. In addition, we took physical, psychological and social factors into consideration, covering a wide variety of diseases and subjective symptoms. Moreover, we used the multiple imputation method to reduce the degree of bias caused by missing values; the results of the sensitivity analysis suggested that factors such as regular hospital visits for COPD may have been overlooked in the complete case analysis.

Several limitations of this study must be noted. First, owing to the cross-sectional design of the study, the causal relationship between LTC and independent variables cannot be determined. Second, certified people may be underrepresented in the self-reported survey. At the time of the survey, $13.0 \%$ of those aged $\geq 65$ years were certified as having care need levels 1-5 [3]. However, only $7.2 \%$ of the participants aged $\geq 65$ years old in this study answered that they were certified for LTC, which is a lower rate than that previously noted despite the fact that people in care facilities, who are thought to account for approximately $30 \%$ of those who are certified [3], were excluded. With a response rate of $79.4 \%$ [10], the participants with LTC certification may have been less likely to have answered the survey. Third, as the survey was based on self-administered questionnaires, the medical diagnoses were not validated by healthcare professionals. Moreover, subjective symptoms and diseases may be underreported, especially in people with dementia, in the self-reported survey. Finally, as people who were admitted to hospitals or care facilities at the time of survey were excluded, those with severe care needs may be underrepresented.

\section{Conclusions}

In conclusion, we identified the factors associated with LTC certification using nationally representative crosssectional data; in addition to physical factors, social and psychological factors were identified. Although causal relationships are yet to be evaluated, multidimensional approaches, including prevention of the progression of lifestyle-related diseases, early intervention regarding mental health-related issues and provision of opportunities for social interactions, may be worth considering to prevent LTC.

\section{Abbreviations}

LTC: Long-term care; ADLs: Activities of daily living; CSLC: Comprehensive Survey of Living Conditions; MHLW: Ministry of Health, Labour and Welfare; ROC: Receiver operating characteristic; AUC: Area under the curve; OR: Odds ratio; Cl: Confidence interval; aOR: Adjusted odds ratios; COPD: Chronic obstructive pulmonary disease

\section{Supplementary Information}

The online version contains supplementary material available at https://doi. org/10.1186/s12877-021-02308-5.

Additional file 1: Supplementary Table 1. Adjusted odds ratios of LTC certification in participants aged $\geq 65$ years in the complete case analysis. LTC, long-term care.

Additional file 2: Supplementary Table 2. Basic characteristics of participants aged $40-64,65-74$ and $\geq 75$ years with or without LTC certification. LTC, long-term care.

Additional file 3: Supplementary Table 3. Non-adjusted odds ratios of LTC certification in participants aged $40-64,65-74$ and $\geq 75$ years. LTC, long-term care.

Additional file 4: Supplementary Table 4. Basic characteristics of certified participants aged $\geq 65$ years with a lower or higher degree of independence in daily life activities.

Additional file 5: Supplementary Table 5. Adjusted odds ratios of factors associated with LTC certification with a lower or higher degree of independence. LTC, long-term care. 
Additional file 6: Supplementary Table 6. Non-adjusted odds ratios of LTC certification among participants aged $\geq 65$ years requiring assistance or supervision due to disabilities or impaired physical function. LTC, long-term care.

\section{Acknowledgements}

\section{Not applicable.}

\section{Authors' contributions}

AM, SY and TK designed the study. AM and SY analysed the data. AM, SY and TK drafted the manuscript. All authors contributed to the interpretation of the data, revised the manuscript and approved the final manuscript. SY and TK contributed equally as corresponding authors.

\section{Funding}

This study was conducted at the Department of Prevention of Diabetes and Lifestyle-Related Diseases, which is engaged in a cooperative program between The University of Tokyo and Asahi Mutual Life Insurance Company, which is the funding organisation for the present study. AM, SY, AO, KIK, DN, HK and TK were members of the department when the study was conducted. AM, KIK and DN are employees of Asahi Mutual Life Insurance Company.

\section{Availability of data and materials}

The data that support the findings of this study are available from MHLW after application for the data is examined and approved.

\section{Declarations}

\section{Ethics approval and consent to participate}

This study was approved by the Institutional Review Board of the University of Tokyo (2018030NI). As the data we obtained were anonymised, no consent was required.

\section{Consent for publication}

Not applicable.

\section{Competing interests}

This study was conducted at the Department of Prevention of Diabetes and Lifestyle-Related Diseases, which is engaged in a cooperative program between the University of Tokyo and Asahi Mutual Life Insurance Company, which is the funding organisation for the present study. AM, SY, AO, KIK, DN, HK and TK were members of the department when the study was conducted. AM, KIK and DN are employees of Asahi Mutual Life Insurance Company.

\section{Author details}

${ }^{1}$ Department of Prevention of Diabetes and Lifestyle-Related Diseases, Graduate School of Medicine, The University of Tokyo, 7-3-1 Hongo, Bunkyo-ku, Tokyo 113-8655, Japan. ²Asahi Mutual Life Insurance Company, Tokyo, Japan. ${ }^{3}$ Department of Pathology and Tumor Biology, Kyoto University, Kyoto, Japan. ${ }^{4}$ Division of Nephrology and Endocrinology, Graduate School of Medicine, The University of Tokyo, Tokyo, Japan. ${ }^{5}$ Department of Diabetes and Metabolic Diseases, Graduate School of Medicine, The University of Tokyo, Tokyo, Japan. ${ }^{6}$ Toranomon Hospital, Tokyo, Japan.

Received: 28 December 2020 Accepted: 28 May 2021

Published online: 21 June 2021

\section{References}

1. Tamiya N, Noguchi H, Nishi A, Reich MR, Ikegami N, Hashimoto H, et al. Population ageing and wellbeing: lessons from Japan's long-term care insurance policy. Lancet. 2011;378:1183-92. https://doi.org/10.1016/S01406736(11)61176-8 PMID 21885099.

2. Iwagami M, Tamiya $\mathrm{N}$. The long-term care insurance system in Japan: past, present, and future. JMA J. 2019;2(1):67-9. https://doi.org/10.31662/jmaj.201 8-0015.
3. Survey of long-term care benefit expenditures. Ministry of Health, Labour and Welfare. Available from: https://www.mhlw.go.jp/toukei/list/45-1b.html. Accessed 27 Apr 2021.

4. Masui $H$, Otaga M, Moriyama Y, Matsushige T. Current issues in long-term care policy and research: toward the promotion of evidence-based policy. Hokeniryokagaku. 2019:68(1):34-44 https://doi.org/10.20683/jniph.68.134.

5. Hoogendijk EO, Afilalo J, Ensrud KE, Kowal P, Onder G, Fried LP. Frailty: implications for clinical practice and public health. Lancet. 2019;394:136575. https://doi.org/10.1016/S0140-6736(19)31786-6 PMID 31609228.

6. Gobbens RJ, Luijkx KG, van Assen MA. Explaining quality of life of older people in the Netherlands using a multidimensional assessment of frailty. Qual Life Res. 2013;22:2051-61. https://doi.org/10.1007/s11136-012-0341-1 PMID 23274572

7. Koller D, Schön G, Schäfer I, Glaeske G, van den Bussche H, Hansen H. Multimorbidity and long-term care dependency--a five-year follow-up. BMC Geriatr. 2014;14:70. https://doi.org/10.1186/1471-2318-14-70 PMID 24884813.

8. Naruse T, Sakai M, Matsumoto H, Nagata S. Diseases that precede disability among latter-stage elderly individuals in Japan. BioSci Trends. 2015;9:270-4. https://doi.org/10.5582/bst.2015.01059 PMID 26355228.

9. Iwagami M, Taniguchi $Y$, Jin X, Adomi M, Mori T, Hamada S, et al. Association between recorded medical diagnoses and incidence of longterm care needs certification: a case control study using linked medical and long-term care data in two Japanese cities. Annals Clin Epidemiol. 2019;1: 56-68. https://doi.org/10.37737/ace.1.2_56.

10. Summary report of Comprehensive Survey of Living Conditions 2013. Ministry of Health, Labour and Welfare. Available from: https://www.mhlw. go.jp/english/database/db-hss/cslc-report2013.html. Accessed 27 Apr 2021.

11. Kondo N, Kazama M, Suzuki K, Yamagata Z. Impact of mental health on daily living activities of Japanese elderly. Prev Med. 2008;46:457-62. https:// doi.org/10.1016/j.ypmed.2007.12.007 PMID 18258290.

12. Monma T, Takeda F, Noguchi H, Tamiya N. Age and sex differences of risk factors of activity limitations in Japanese older adults. Geriatr Gerontol Int. 2016;16:670-8. https://doi.org/10.1111/ggi.12533 PMID 26044713.

13. Paterniti S, Verdier-Taillefer MH, Dufouil C, Alpérovitch A. Depressive symptoms and cognitive decline in elderly people. Longitudinal study. Br J Psychiatry. 2002;181:406-10. https://doi.org/10.1192/bjp.181.5.406 PMID 12411266.

14. Schnitzer S, Blüher S, Teti A, Schaeffner E, Ebert N, Martus $P$, et al. Risk profiles for care dependency: cross-sectional findings of a population-based cohort study in Germany. J Aging Health. 2020;32:352-60. https://doi.org/1 0.1177/0898264318822364 PMID 30658538.

15. Hsu WC, Wang JY, Tsai AC. Predictors of developing a new need for longterm care of older adults aged $\geq 70$ years: results from a population-based cohort study in Taiwan. Geriatr Gerontol Int. 2019;19:641-6. https://doi.org/1 0.1111/ggi.13684 PMID 31099138.

16. Wu CY, Hu HY, Huang N, Fang YT, Chou YJ, Li CP. Determinants of longterm care services among the elderly: a population-based study in Taiwan. PLoS One. 2014;9:e89213. https://doi.org/10.1371/journal.pone.0089213 PMID 24586602

17. Moriya S, Murata A, Kimura S, Inoue N, Miura H. Predictors of eligibility for long-term care funding for older people in Japan. Australas J Ageing. 2013; 32:79-85. https://doi.org/10.1111/j.1741-6612.2012.00601.x PMID 23773245.

18. Comprehensive Survey of Living Conditions. Ministry of Health, Labour and Welfare. Available from: https://www.mhlw.go.jp/english/database/db-hss/ cslc.html. Accessed 27 Apr 2021.

19. Andersen R, Newman JF. Societal and individual determinants of medical care utilization in the United States. Milbank Mem Fund Q Health Soc. 1973: 51(1):95-124. https://doi.org/10.1111/j.1468-0009.2005.00428.x PMID: 4198894.

20. Kessler RC, Andrews G, Colpe LJ, Hiripi E, Mroczek DK, Normand SL, et al. Short screening scales to monitor population prevalences and trends in non-specific psychological distress. Psychol Med. 2002;32:959-76. https://doi. org/10.1017/s0033291702006074 PMID 12214795.

21. van Buuren S, Groothuis-Oudshoorn K. Mice: multivariate imputation by chained equations in R. J Stat Softw. 2011;45(3):1-67. https://doi.org/10.1863 7/jss.v045.i03.

22. Kiyoshige E, Kabayama M, Gondo Y, Masui Y, Ryuno H, Sawayama Y, et al. Association between long-term care and chronic and lifestyle-related disease modified by social profiles in community-dwelling people aged 80 and 90; SONIC study. Arch Gerontol Geriatr. 2019;81:176-81. https://doi. org/10.1016/j.archger.2018.12.010 PMID 30597340. 
23. Results of the National Health and Nutrition Survey Japan; 2013. Available from: https://www.mhlw.go.jp/bunya/kenkou/eiyou/h25-houkoku.html. Accessed 27 Apr 2021.

24. Gale CR, Cooper C, Deary IJ, Aihie SA. Psychological well-being and incident frailty in men and women: the English Longitudinal Study of Ageing. Psychol Med. 2014;44:697-706. https://doi.org/10.1017/S0033291713001384 PMID 23822897.

25. Penninx BW, Leveille S, Ferrucci L, van Eijk JT, Guralnik JM. Exploring the effect of depression on physical disability: Iongitudinal evidence from the established populations for epidemiologic studies of the elderly. Am J Public Health. 1999:89:1346-52. https://doi.org/10.2105/ajph.89.9.1346 PMID 10474551 .

26. Stuck AE, Walthert JM, Nikolaus T, Büla CJ, Hohmann C, Beck JC. Risk factors for functional status decline in community-living elderly people: a systematic literature review. Soc Sci Med. 1999;48:445-69. https://doi.org/1 0.1016/s0277-9536(98)00370-0 PMID 10075171.

27. Ramsay SE, Whincup PH, Morris RW, Lennon LT, Wannamethee SG. Extent of social inequalities in disability in the elderly: results from a population-based study of British men. Ann Epidemiol. 2008;18:896-903. https://doi.org/10.101 6/j.annepidem.2008.09.006 PMID 19041588.

\section{Publisher's Note}

Springer Nature remains neutral with regard to jurisdictional claims in published maps and institutional affiliations.

Ready to submit your research? Choose BMC and benefit from:

- fast, convenient online submission

- thorough peer review by experienced researchers in your field

- rapid publication on acceptance

- support for research data, including large and complex data types

- gold Open Access which fosters wider collaboration and increased citations

- maximum visibility for your research: over $100 \mathrm{M}$ website views per year

At BMC, research is always in progress.

Learn more biomedcentral.com/submissions 